\title{
Article \\ Calderón Operator on Local Morrey Spaces with Variable Exponents
}

\author{
Kwok-Pun Ho (D) \\ Department of Mathematics and Information Technology, The Education University of Hong Kong, \\ 10 Lo Ping Road, Tai Po, Hong Kong, China; vkpho@eduhk.hk
}

check for updates

Citation: Ho, K.-P. Calderón Operator on Local Morrey Spaces with Variable Exponents. Mathematics 2021, 9, 2977. https://doi.org/ $10.3390 /$ math 9222977

Academic Editor: Palle E. T. Jorgensen

Received: 20 October 2021

Accepted: 18 November 2021

Published: 22 November 2021

Publisher's Note: MDPI stays neutral with regard to jurisdictional claims in published maps and institutional affiliations.

Copyright: (C) 2021 by the authors. Licensee MDPI, Basel, Switzerland. This article is an open access article distributed under the terms and conditions of the Creative Commons Attribution (CC BY) license (https:// creativecommons.org/licenses/by/ $4.0 /)$.

\begin{abstract}
In this paper, we establish the boundedness of the Calderón operator on local Morrey spaces with variable exponents. We obtain our result by extending the extrapolation theory of Rubio de Francia to the local Morrey spaces with variable exponents. The exponent functions of the local Morrey spaces with the exponent functions are only required to satisfy the log-Hölder continuity assumption at the origin and infinity only. As special cases of the main result, we have Hardy's inequalities, the Hilbert inequalities and the boundedness of the Riemann-Liouville and Weyl averaging operators on local Morrey spaces with variable exponents.
\end{abstract}

Keywords: Calderón operator; Hardy's inequality; variable Lebesgue space; local Morrey space; local block space; extrapolation

\section{Introduction}

The main theme of this paper is the boundedness of the Calderón operator on local Morrey spaces with variable exponents.

The Calderón operator is one of the important operators in harmonic analysis and theory of function spaces. The Calderón operator is related with the Hardy' inequality, the Stieltjes transformation, the Riemann-Liouville and Weyl averaging operators. It also gives an estimate for the maximal Hilbert transform ([1], Chapter 3, Theorem 4.7). Moreover, the boundedness of the Calderón operator is also related with the convergence of Fourier series on rearrangement-invariant Banach function spaces ([1], Chapter 3, Theorem 6.10).

The boundedness of the Calderón operator on Lebesgue spaces is a well known result [2]. Recently, the boundedness property has been extended to the weighted Lebesgue spaces [3] and the weighted Lebesgue spaces with variable exponents [4]. In this paper, we further extend the boundedness of the Calderón operator to local Morrey spaces with variable exponents.

The local Morrey spaces with variable exponents are extensions of the classical Morrey spaces introduced and studied by Morrey [5] and the Lebesgue spaces with variable exponents [6,7]. The mapping properties of singular integral operators, the fractional integral operators, the geometric maximal operators and the spherical maximal functions were obtained in [8-14].

In this paper, we obtain our main results by extending the techniques from the extrapolation theory introduced by Rubio de Francia [15-17] to local Morrey spaces with variable exponents. An extrapolation theory for local Morrey spaces with variable exponents was obtained in [14], while the extrapolation theory given in [14] is based on the Hardy-Littlewood maximal function. In this paper, we use another maximal function from [3] which is defined via the basis $\{(0, r): r>0\}$. Similar to the results in [4], by using this maximal function, the exponent functions for the local Morrey spaces with variable exponents is not required to be globally log-Hölder continuous function. The exponent function is just required to be log-Hölder continuous at origin and infinity.

This paper is organized as follows. The definition and the boundedness of the Calderón operator on weighted Lebesgue spaces were presented in Section 2. The definitions of local 
Morrey spaces with variable exponents and local block spaces with variable exponents are given in Section 3. The local block spaces with variable exponents are pre-duals of local Morrey spaces with variable exponents, and the boundedness of the maximal function associated with the the basis $\{(0, r): r>0\}$ on the local block spaces with variable exponents is obtained in Section 3. This boundedness result is one of the crucial results for the boundedness of the Calderón operator obtained in Section 4. As applications of our main results, we obtain the Hardy' inequalities, the boundedness of the Stieltjes transformation, the Riemann-Liouville and Weyl averaging operators on local Morrey spaces with variable exponents.

\section{Definitions and Preliminaries}

Let $\mathcal{M}$ be the class of Lebesgue measurable functions on $(0, \infty)$.

For any non-negative function $f$ on $(0, \infty)$, the Calderón operator is defined as

$$
S f(x)=\frac{1}{x} \int_{0}^{x} f(y) d y+\int_{x}^{\infty} \frac{f(y)}{y} d y, \quad x \in(0, \infty) .
$$

For any non-negative function $f$ on $(0, \infty)$, the Hardy operator is defined as

$$
\mathcal{H} f(x)=\frac{1}{x} \int_{0}^{x} f(y) d y, \quad x \in(0, \infty) .
$$

We see that the adjoint operator of $\mathcal{H}$ is given by

$$
\mathcal{H}^{*} f(x)=\int_{x}^{\infty} \frac{f(y)}{y} d y, \quad x \in(0, \infty) .
$$

The boundedness of $\mathcal{H}$ and $\mathcal{H}^{*}$ on Lebesgue spaces is called the Hardy's inequalities. We see that $S=\mathcal{H}+\mathcal{H}^{*}$. Thus, the boundedness of the Calderón operator on Lebesgue spaces follow from the Hardy's inequalities. The reader is referred to $[2,18,19]$ for the studies of Hardy's inequalities.

Let $\alpha \geq 0$; the Stieltjes transformation, the Riemann-Liouville and Weyl averaging operators are defined as

$$
\begin{aligned}
& H f(x)=\int_{0}^{\infty} \frac{f(y)}{x+y} d y \\
& I_{\alpha} f(x)=\frac{\alpha+1}{x^{\alpha+1}} \int_{0}^{x}(x-y)^{\alpha} f(y) d y, \\
& J_{\alpha} f(x)=(\alpha+1) \int_{x}^{\infty} \frac{(y-x)^{\alpha}}{y^{\alpha+1}} f(y) d y .
\end{aligned}
$$

For any non-negative function $f$, we have $H f(x) \leq S f(x), I_{\alpha} f(x) \leq S f(x)$ and $J_{\alpha} f(x) \leq S f(x)$. The reader is referred to [20-22] for the studies of the Stieltjes transformation and its application on the Hilbert's double series.

We recall the following maximal operator and the Muckenhoupt type classes of weight functions for $S$. They were introduced in [3]. For any locally integrable function $f$, define

$$
N f(x)=\sup _{b>x} \frac{1}{b} \int_{0}^{b}|f(y)| d y, \quad x>0 .
$$

The operator $N$ is the maximal operator on $(0, \infty)$ with the basis $\{(0, r): r>0\}$. Notice that for any non-negative function $f$, we have $N f \leq S f$.

We recall the following class of weighted functions from ([3], (1.2)). 
Definition 1. Let $p \in(1, \infty)$. We say that a Lebesgue measurable function $\omega:(0, \infty) \rightarrow[0, \infty)$ belongs to $A_{p, 0}$ if

$$
\sup _{b>0}\left(\frac{1}{b} \int_{0}^{b} \omega(x) d x\right)\left(\frac{1}{b} \int_{0}^{b} \omega(x)^{1-p^{\prime}} d x\right)^{p-1}<\infty
$$

where $p^{\prime}$ is the conjugate of $p$.

The class $A_{1,0}$ consists of all Lebesgue measurable function $\omega:(0, \infty) \rightarrow[0, \infty)$ satisfying

$$
N \omega(x) \leq C \omega(x), \quad x \in(0, \infty)
$$

and $[w]_{A_{1,0}}$ denotes the smallest constant for which the above inequality holds.

In view of ([3], Theorem 1.1), we have the following weighted norm inequalities for $N$.

Theorem 1. Let $p \in(1, \infty)$. We have a constant $C>0$ such that

$$
\int_{0}^{\infty}|N f(x)|^{p} \omega(x) d x \leq C \int_{0}^{\infty}|f(x)|^{p} \omega(x) d x
$$

if and only if $\omega \in A_{p, 0}$.

When $p \in(1, \infty)$, the class $A_{p, 0}$ coincides with the class $\mathcal{C}_{p}$ introduced in [23]; see ([3], Theorem 1.2). In addition, as a special case of ([3], Theorem 1.2), we have the weighted norm inequalities for the Calderón operator.

Theorem 2. Let $p \in(1, \infty)$. We have a constant $C>0$ such that

$$
\int_{0}^{\infty}|S f(x)|^{p} \omega(x) d x \leq C \int_{0}^{\infty}|f(x)|^{p} \omega(x) d x
$$

if and only if $\omega \in A_{p, 0}$.

\section{Local Morrey Spaces with Variable Exponents}

In this section, we recall the definition of local Morrey space with variable exponent and study a pre-dual of this space, namely, the local block space with variable exponent. As a crucial supporting result for our main result, we obtain the boundedness of the maximal function $N$ on local block spaces with variable exponents at the end of this section.

We recall the definition of Lebesgue spaces with variable exponents.

Definition 2. Let $p(\cdot):(0, \infty) \rightarrow[1, \infty)$ be a Lebesgue measurable function. The Lebesgue space with variable exponent $L^{p(\cdot)}$ consists of all Lebesgue measurable functions $f:(0, \infty) \rightarrow \mathbb{C}$ satisfying

$$
\|f\|_{L^{p(\cdot)}}=\inf \left\{\lambda>0: \rho_{p(\cdot)}(f / \lambda) \leq 1\right\}<\infty
$$

where

$$
\rho_{p(\cdot)}(f)=\int_{0}^{\infty}|f(x)|^{p(x)} d x .
$$

We call $p(x)$ the exponent function of $L^{p(\cdot)}$.

Let $p^{\prime}(x)$ be the conjugate function of $p(x)$. That is, they satisfy $\frac{1}{p(x)}+\frac{1}{p^{\prime}(x)}=1$,

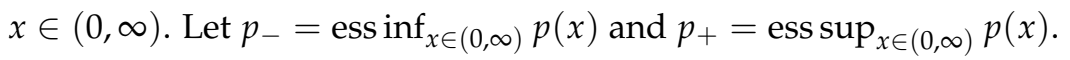


Definition 3. A continuous function $g$ on $(0, \infty)$ is log-Hölder continuous at the origin if there exist $c_{\log }>0$ and $g_{0}$ such that

$$
\left|g(x)-g_{0}\right| \leq \frac{c_{\log }}{-\log (x)}, \quad \forall x \in(0,1 / 2)
$$
so that

A continuous function is log-Hölder continuous at infinity if there exist $g_{\infty} \in \mathbb{R}$ and $c_{\infty}>0$

$$
\left|g(x)-g_{\infty}\right| \leq \frac{c_{\infty}}{\log (e+|x|)}, \quad \forall x \in(0, \infty) .
$$

We write $g \in C^{\log }$ if $g$ is log-Hölder continuous at origin and log-Hölder continuous at infinity.

The above classes of log-Hölder continuous functions are used in [24-26] for the studies of Herz spaces with variable exponents.

We have the boundedness of the maximal operator $N$ on $L^{p(\cdot)}$ whenever $p(\cdot) \in C^{\log }$ with $1<p_{-} \leq p_{+}<\infty$.

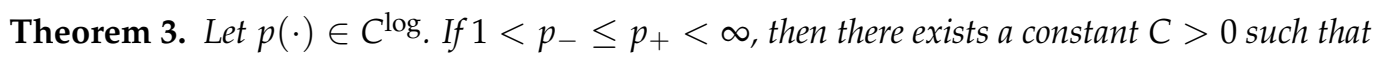

$$
\|N f\|_{L^{p(\cdot)}} \leq C\|f\|_{L^{p(\cdot)}} .
$$

For the proof of the above theorem, the reader is referred to ([4], Theorem 1.6 and Section 3).

We now give the definitions of local Morrey spaces with variable exponents from [14].

Definition 4. Let $p(\cdot):(0, \infty) \rightarrow(1, \infty)$ and $u:(0, \infty) \rightarrow(0, \infty)$ be Lebesgue measurable functions. The local Morrey space with variable exponent $L M_{u}^{p(\cdot)}$ consists of all $f \in \mathcal{M}$ satisfying

$$
\|f\|_{L M_{u}^{p(\cdot)}}=\sup _{r>0} \frac{1}{u(r)}\left\|\chi_{(0, r)} f\right\|_{L^{p(\cdot)}}<\infty
$$

When $p(\cdot)=p, 1 \leq p<\infty$, the local Morrey space with variable exponent becomes the local Morrey space $L M_{u}^{p}$. For the studies of local Morrey spaces, the reader is referred to [9-13]. For the mapping properties of the Carleson operator, the local sharp maximal functions, the geometrical maximal functions and the rough maximal functions on $L M_{u}^{p(\cdot)}$, see $[14,27]$.

The local Morrey spaces with variable exponents are ball Banach function spaces defined and studied in [28,29]; see the discussion after ([27], Theorem 2.3).

We recall a class of weight functions for the studies of the local Morrey spaces with variable exponents defined in ([14], Definition 2.5).

Definition 5. Let $q_{0} \in(0, \infty), p(\cdot):(0, \infty) \rightarrow[1, \infty]$. We say that a Lebesgue measurable function, $u(r):(0, \infty) \rightarrow(0, \infty)$, belongs to $\mathbb{L} \mathbb{W}_{p(\cdot)}^{q_{0}}$ if there exists a constant $C>0$ such that for any $r>0$, u fulfills

$$
\begin{aligned}
& C \leq u(r), \quad \forall r \geq 1, \\
& \left\|\chi_{(0, r)}\right\|_{L^{p(\cdot)}} \leq C u(r), \quad \forall r<1, \\
& \sum_{j=0}^{\infty} \frac{\left\|\chi_{(0, r)}\right\|_{L^{p(\cdot) / q_{0}}}}{\left\|\chi_{\left(0,2^{j+1} r\right)}\right\|_{L^{p(\cdot) / q_{0}}}}\left(u\left(2^{j+1} r\right)\right)^{q_{0}}<C(u(r))^{q_{0}}
\end{aligned}
$$

for all $r>0$. 


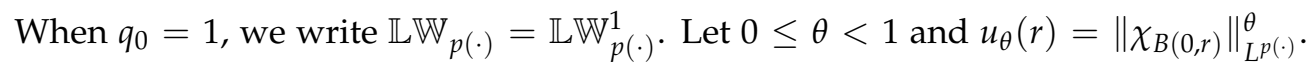
The discussion at the end of ([30], Section 2) shows that $u_{\theta} \in \mathbb{L}_{p}(\cdot)$. Particularly, $u \equiv 1$ is a member of $\mathbb{L} \mathbb{W}_{p(\cdot)}$.

Next, we recall a pre-dual of the local Morrey space with variable exponent from ([14], Definition 3.1).

Definition 6. Let $p(\cdot):(0, \infty) \rightarrow(0, \infty)$ and $u(r):(0, \infty) \rightarrow(0, \infty)$ be Lebesgue measurable functions. $A b \in \mathcal{M}$ is a local $\left(u, L^{p(\cdot)}\right)$-block if it is supported in $(0, r), r>0$ and

$$
\|b\|_{L^{p(\cdot)}} \leq \frac{1}{u(r)}
$$

We write $b \in \mathfrak{l b}_{u, L^{p(\cdot)}}$ if $b$ is a local $\left(u, L^{p(\cdot)}\right)$-block.

Define $\mathfrak{L}^{\mathfrak{B}_{u, p(\cdot)}}$ by

$$
\mathfrak{L B}_{u, p(\cdot)}=\left\{\sum_{k=1}^{\infty} \lambda_{k} b_{k}: \sum_{k=1}^{\infty}\left|\lambda_{k}\right|<\infty \text { and } b_{k} \text { is a local }\left(u, L^{p(\cdot)}\right) \text {-block }\right\} .
$$

The space $\mathfrak{L} \mathfrak{B}_{u, p(\cdot)}$ is endowed with the norm

$$
\|f\|_{\mathfrak{L B}_{u, p(\cdot)}}=\inf \left\{\sum_{k=1}^{\infty}\left|\lambda_{k}\right| \text { such that } f=\sum_{k=1}^{\infty} \lambda_{k} b_{k} \text { a.e. }\right\} .
$$

We call $\mathfrak{L B}_{u, p(\cdot)}$ the local block space with variable exponent.

In view of ([14], Theorem 3.3), $\mathfrak{L} \mathfrak{B}_{u, p(\cdot)}$ is a Banach space and $\mathfrak{L} \mathfrak{B}_{u, p(\cdot)} \subset L_{\text {loc }}^{1}$. In addition, whenever $f, g \in \mathcal{M}$ satisfying $|f| \leq|g|$ and $g \in \mathfrak{L} \mathfrak{B}_{u, p(\cdot)}$, we have $f \in \mathfrak{L} \mathfrak{B}_{u, p(\cdot)}$ ([14], Proposition 3.2).

We present the following results for the block spaces with variable exponent from ([14], Section 3). Notice that the results in [14] are for local Morrey spaces with variable exponents on $\mathbb{R}^{n}$, while with some simple modifications, the results and the proofs in [14] can be extended to local Morrey spaces with variable exponents on $(0, \infty)$.

Theorem 4. Let $p(\cdot):(0, \infty) \rightarrow(1, \infty)$ and $u:(0, \infty) \rightarrow(0, \infty)$ be Lebesgue measurable functions. We have

$$
\mathfrak{L} \mathfrak{B}_{u, p(\cdot)}^{*}=L M_{u}^{p^{\prime}(\cdot)}
$$

where $\mathfrak{L B}_{u, p(\cdot)}^{*}$ denotes the dual space of $\mathfrak{L} \mathfrak{B}_{u, p(\cdot)}$.

The reader is referred to ([14], Theorem 3.1) for the proof of the above results. Furthermore, the proof of ([14], Theorem 3.1) gives the Hölder inequalities for $f \in L M_{u}^{p^{\prime}(\cdot)}$ and $g \in \mathfrak{L} \mathfrak{B}_{u, p(\cdot)}$

$$
\int_{0}^{\infty}|f(x) g(x)| d x \leq C\|g\|_{\mathfrak{L}^{\mathfrak{B}_{u, p(\cdot)}}}\|f\|_{L M_{u}^{p^{\prime}(\cdot)}}
$$

for some $C>0$.

Moreover, in the proof of ([14], Theorem 3.1), we also have the norm conjugate formula

$$
C_{0}\|f\|_{L M_{u}^{p(\cdot)}} \leq \sup _{h \in \mathfrak{l b}_{u, p(\cdot)}} \int_{0}^{\infty}|f(x) h(x)| d x \leq C_{1}\|f\|_{L M_{u}^{p(\cdot)}}
$$

for some $C_{0}, C_{1}>0$.

Proposition 1. Let $p(\cdot):(0, \infty) \rightarrow(1, \infty), u:(0, \infty) \rightarrow(0, \infty)$ be Lebesgue measurable functions and $f \in \mathfrak{L B}_{u, p(\cdot)}$. If $g \in \mathcal{M}$ satisfying $|g| \leq|f|$, then $g \in \mathfrak{L B}_{u, p(\cdot)}$. 
The proof of the preceding proposition is given in ([14], Proposition 3.2.). We establish a supporting lemma in the following paragraphs.

Lemma 1. Let $p(\cdot) \in C^{\log }$ with $1<p_{-} \leq p_{+}<\infty$. We have constants $C_{0}, C_{1}>0$ such that for any $r>0$, we have

$$
C_{0} r \leq\left\|\chi_{(0, r)}\right\|_{L^{p^{\prime}(\cdot)}}\left\|\chi_{(0, r)}\right\|_{L^{p(\cdot)}} \leq C_{1} r
$$

Proof. The first inequality in (12) follows from the Hölder inequality for Lebesgue spaces with variable exponents.

For any $r>0$ and locally integrable function $f$, define

$$
P_{r} f(y)=\left(\frac{1}{r} \int_{0}^{r} f(x) d x\right) \chi_{(0, r)}(y)
$$

The definition of $N$ guarantees that $\left|P_{r} f\right| \leq N f$. Therefore, we have $\left\|P_{r}\right\|_{L^{p(\cdot)} \rightarrow L^{p(\cdot)}} \leq$ $\|N\|_{L^{p(\cdot)} \rightarrow L^{p(\cdot)}}$. According to ([7], Corollary 3.2.14), we have

$$
\left\|\chi_{(0, r)}\right\|_{L^{p^{\prime}(\cdot)}}\left\|\chi_{(0, r)}\right\|_{L^{p(\cdot)}}=\sup \left\{\left|\int_{0}^{r} g(x) d x\right|\left\|\chi_{(0, r)}\right\|_{L^{p(\cdot)}}:\|g\|_{L^{p(\cdot)}} \leq 1\right\} .
$$

Theorem 3 yields a constant $C_{1}>0$ such that for any $r>0$, we have

$$
\begin{aligned}
\left\|\chi_{(0, r)}\right\|_{L^{p^{(}(\cdot)}}\left\|\chi_{(0, r)}\right\|_{L^{p(\cdot)}} & \leq \sup \left\{r\left\|P_{r} g\right\|_{L^{p(\cdot)}}:\|g\|_{L^{p(\cdot)}} \leq 1\right\} \\
& \leq \sup \left\{r\|N g\|_{L^{p(\cdot)}}:\|g\|_{L^{p(\cdot)}} \leq 1\right\} \leq C_{1} r .
\end{aligned}
$$

Therefore, the second inequality in (12) holds.

We are now ready to obtain the boundedness of the maximal function $N$ on $\mathfrak{L}_{u, p(\cdot)}$.

Theorem 5. Let $p(\cdot):(0, \infty) \rightarrow(1, \infty)$ and $u:(0, \infty) \rightarrow(0, \infty)$ be Lebesgue measurable functions. If $p(\cdot) \in C^{\log }$ with $1<p_{-} \leq p_{+}<\infty$ and $u \in \mathbb{L} \mathbb{W}_{p^{\prime}(\cdot)}$, then the maximal operator $N$ is bounded on $\mathfrak{L}_{u, p(\cdot)}$.

Proof. In view of ([14], Theorem 3.3), we have $\mathfrak{L}_{\mathfrak{B}_{u, p}(\cdot)} \subset L_{\text {loc }}^{1}$; therefore, the maximal operator $N$ is well defined on $\mathfrak{L} \mathfrak{B}_{u, p(\cdot)}$.

Let $b \in \mathfrak{l b}_{u, L^{p(\cdot)}}$ with support $(0, r), r>0$. For any $k \in \mathbb{N}$, write $B_{k}=\left(0,2^{k} r\right)$. Define $n_{k}=\chi_{B_{k+1} \backslash B_{k}} N b, k \in \mathbb{N} \backslash\{0\}$ and $n_{0}=\chi_{(0,2 r)} N b$. We have supp $n_{k} \subseteq B_{k+1} \backslash B_{k}$ and $\mathrm{Nb}=\sum_{k=0}^{\infty} n_{k}$.

As $p(\cdot) \in C^{\log }$ with $1<p_{-} \leq p_{+}<\infty$, Theorem 3 guarantees that

$$
\left\|n_{0}\right\|_{L^{p(\cdot)}} \leq C\|N b\|_{L^{p(\cdot)}} \leq \frac{C}{u(r)} \leq \frac{C}{u(2 r)}
$$

for some constant $C>0$ independent $r$. The last inequality holds since (6) asserts that $\frac{\left\|\chi_{B(0, r)}\right\|_{L^{p(\cdot)}}}{\left\|\chi_{B(0,2 r)}\right\|_{L^{p(\cdot)}}} u(2 r) \leq C u(r)$ and ([4], Lemma 2.3) yields $\left\|\chi_{B(0,2 r)}\right\|_{L^{p(\cdot)}} \leq C\left\|\chi_{B(0, r)}\right\|_{L^{p(\cdot)}}$ for some $C>0$ independent of $r>0$. As a result of the above inequalities, $n_{0}$ is a constant-multiple of a local $\left(u, L^{p(\cdot)}\right)$-block.

The Hölder inequality for $L^{p(\cdot)}$ yields

$$
\begin{aligned}
n_{k}=\chi_{B_{k+1} \backslash B_{k}} N b & \leq \frac{\chi_{B_{k+1} \backslash B_{k}}}{2^{k} r} \int_{0}^{r}|b(x)| d x \\
& \leq C \chi_{B_{k+1} \backslash B_{k}} \frac{1}{2^{k_{r}}}\|b\|_{L^{p(\cdot)}}\left\|\chi_{(0, r)}\right\|_{L^{p^{\prime}(\cdot)}}
\end{aligned}
$$

for some $C>0$ independent of $k$. 
Consequently, (12) gives

$$
\begin{aligned}
\left\|n_{k}\right\|_{L^{p(\cdot)}} & \leq \frac{\left\|\chi_{B_{k+1} \backslash B_{k}}\right\|_{L^{p(\cdot)}}}{2^{k} r}\|b\|_{L^{p(\cdot)}}\left\|\chi_{B(0, r)}\right\|_{L^{p^{\prime}(\cdot)}} \\
& \leq C \frac{\left\|\chi_{(0, r)}\right\|_{L^{p^{\prime}(\cdot)}}}{\left\|\chi_{B_{k+1}}\right\|_{L^{p^{\prime}(\cdot)}}} \frac{u\left(2^{k+1} r\right)}{u(r)} \frac{1}{u\left(2^{k+1} r\right)} .
\end{aligned}
$$

Write $n_{k}=\sigma_{k} d_{k}$, where

$$
\sigma_{k}=\frac{\left\|\chi_{(0, r)}\right\|_{L^{p^{\prime}(\cdot)}}}{\left\|\chi_{B_{k+1}}\right\|_{L^{p^{\prime}(\cdot)}}} \frac{u\left(2^{k+1} r\right)}{u(r)}
$$

We find that $d_{k}$ is a constant-multiple of a local $\left(u, L^{p(\cdot)}\right)$-block, and this constant does

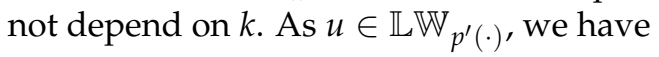

$$
\sum_{j=0}^{\infty} \frac{\left\|\chi_{(0, r)}\right\|_{L^{p^{\prime}(\cdot)}}}{\left\|\chi_{\left(0,2^{j+1} r\right)}\right\|_{L^{p^{\prime}(\cdot)}}} u\left(2^{j+1} r\right) \leq C u(r)
$$

We have $\sum_{k=0}^{\infty} \sigma_{k}<C$ for some $C>0$. Hence, $N b \in \mathfrak{L}^{\mathfrak{B}_{u, p(\cdot)}}$. Moreover, there exists a constant $C_{0}>0$ so that for any local $\left(u, L^{p(\cdot)}\right)$-block $b$,

$$
\|N b\|_{\mathfrak{L}_{\mathfrak{B}_{u, p(\cdot)}}<C_{0}}
$$

Let $f \in \mathfrak{L B}_{u, p(\cdot)}$. The definition of $\mathfrak{L B}_{u, p(\cdot)}$ yields a family of local $\left(u, L^{p(\cdot)}\right)$-blocks $\left\{c_{k}\right\}_{k=1}^{\infty}$ and a sequence $\Lambda=\left\{\lambda_{k}\right\}_{k=1}^{\infty} \in l^{1}$ such that $f=\sum_{k=1}^{\infty} \lambda_{k} c_{k}$ with $\|\Lambda\|_{l^{1}} \leq$ $2\|f\|_{\mathfrak{L} \mathfrak{B}_{u, p(\cdot)}}$. Since $N$ is sublinear, we find that

$$
\begin{aligned}
\left\|\sum_{k=1}^{\infty} \lambda_{k} N c_{k}\right\|_{\mathfrak{L B}_{u, p(\cdot)}} & \leq \sum_{k=1}^{\infty}\left|\lambda_{k}\right|\left\|N c_{k}\right\|_{\mathfrak{L}^{2} \mathfrak{B}_{u, p(\cdot)}} \\
& \leq C_{0} \sum_{k=1}^{\infty}\left|\lambda_{k}\right| \leq 2 C_{0}\|f\|_{\mathfrak{L} \mathfrak{B}_{u, p(\cdot)}} .
\end{aligned}
$$

As $N f \leq \sum_{k=1}^{\infty}\left|\lambda_{k}\right| N c_{k}$, Proposition 1 guarantees that $N f \in \mathfrak{L} \mathfrak{B}_{u, p(\cdot)}$ and $\|N f\|_{\mathfrak{L B}_{u, p(\cdot)}}$ $\leq C\|f\|_{\mathfrak{L} \mathfrak{B}_{u, p(\cdot)}}$ for some $C>0$.

\section{Calderón Operator}

The boundedness of the Calderón operator on local Morrey spaces with variable exponents is established in this section. As applications of our main result, we obtain the Hardy's inequalities and the Hilbert inequalities on local Morrey spaces with variable exponents.

We use the techniques from the extrapolation theory. We first recall an operator from the Rubio de Francia algorithm. Let $p_{0} \in(0, \infty)$ and $p(\cdot) \in C^{\log }$ with $p_{0}<p_{-} \leq p_{+}<\infty$. The operator $\mathcal{R}$ is defined by

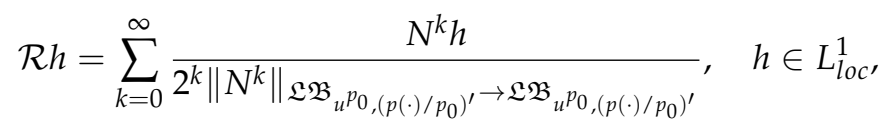

where $N^{k}$ is the $k$ iterations of the operator $N$ and $N^{0} h=|h|$. The following are the boundedness of $N$ and $\mathcal{R}$ on the local block spaces with variable exponents. 
Proposition 2. Let $p_{0} \in(0, \infty)$ and $p(\cdot) \in C^{\log \text { with }} p_{0}<p_{-} \leq p_{+}<\infty$. If $u \in \mathbb{L} \mathbb{W}_{p(\cdot)^{\prime}}^{p_{0}}$ then the operator $\mathcal{R}$ is well defined on $\mathfrak{L}_{\mathfrak{B}^{p_{0}},\left(p(\cdot) / p_{0}\right)^{\prime}}$ and there is a constant $C>0$ such that for any $h \in \mathfrak{L}^{u^{p_{0}},\left(p(\cdot) / p_{0}\right)^{\prime} \text {, }}$

$$
\begin{aligned}
& |h(x)| \leq \mathcal{R} h(x)
\end{aligned}
$$

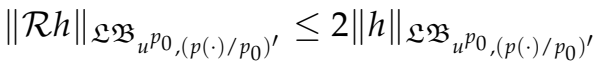

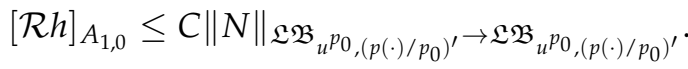

Proof. As $u \in \mathbb{L}_{p(\cdot)}^{p_{0}}$ implies $u^{p_{0}} \in \mathbb{L} \mathbb{W}_{p(\cdot) / p_{0}}$, Theorem 5 guarantees that the maximal operator $N$ is bounded on $\mathfrak{L B}_{u^{p_{0}},\left(p(\cdot) / p_{0}\right)^{\prime}}$. Consequently, the operator $\mathcal{R}$ is well defined

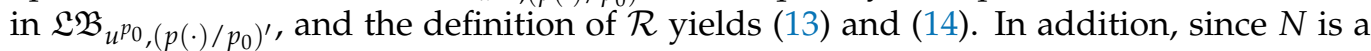
sublinear operator, for any $h \in \mathfrak{L}_{\mathfrak{u}^{p_{0}},\left(p(\cdot) / p_{0}\right)^{\prime}}$, we obtain

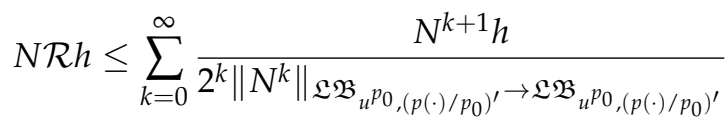

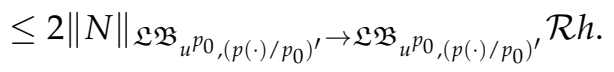

According to Definition $1, \mathcal{R} h \in A_{1,0}$, and hence, (15) holds.

Theorem 6. Let $p(\cdot) \in C^{\log }$ with $1<p_{-} \leq p_{+}<\infty$. If there exists a $p_{0} \in\left(0, p_{-}\right)$such that $u \in \mathbb{L} \mathbb{W}_{p(\cdot)}^{p_{0}}$, then the Calderón operator $S$ is bounded on $L M_{u}^{p(\cdot)}$.

Proof. Let $f \in L M_{u}^{p(\cdot)}$. For any $h \in \mathfrak{L B}_{u^{p_{0}},\left(p(\cdot) / p_{0}\right)^{\prime}},(10)$ and (14) yield

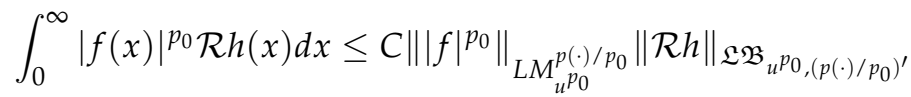

$$
\begin{aligned}
& \leq\|f\|_{L M_{u}^{p(\cdot)}}\|h\|_{\mathfrak{L B}_{\mathfrak{B}^{p}}{ }_{0,\left(p(\cdot) / p_{0}\right)^{\prime}}} .
\end{aligned}
$$

Thus, we have

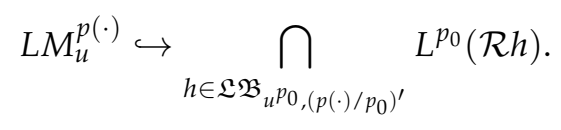

Theorem 4 guarantees

$$
\begin{aligned}
& \|S f\|_{L M_{u}^{p(\cdot)}}^{p_{0}}=\left\||S f|^{p_{0}}\right\|_{L M_{u}^{p(\cdot) / p_{0}}} \\
& \leq C \sup \left\{\int_{0}^{\infty}|S f(x)|^{p_{0}}|h(x)| d x:\|h\|_{\mathcal{L B}_{u^{p}} p_{0,\left(p(\cdot) / p_{0}\right)^{\prime}}} \leq 1\right\}
\end{aligned}
$$

for some $C>0$.

In view of (15), $\mathcal{R} h \in A_{1,0}$. Furthermore, the embedding (16) guarantees that (1) holds for all $f \in L M_{u}^{p(\cdot)}$. Consequently, by applying $\omega=\mathcal{R} h$ on (1) and using (13), we find that

$$
\begin{aligned}
\int_{0}^{\infty}|S f(x)|^{p_{0}} h(x) d x & \leq \int_{0}^{\infty}|S f(x)|^{p_{0}} \mathcal{R} h(x) d x \\
& \leq C \int_{0}^{\infty}|f(x)|^{p_{0}} \mathcal{R} h(x) d x .
\end{aligned}
$$


Consequently, (10) and (14) give

$$
\begin{aligned}
& \int_{0}^{\infty}|S f(x)|^{p_{0}} h(x) d x \leq C\left\||f|^{p_{0}}\right\|_{L M_{u^{p}}^{p(\cdot) / p_{0}}}\|\mathcal{R} h\|_{\mathfrak{L}_{\mathfrak{B}_{u}} p_{0,\left(p(\cdot) / p_{0}\right)^{\prime}}}
\end{aligned}
$$

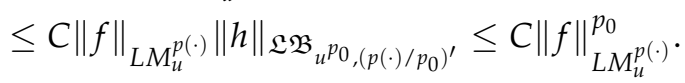

By taking supremum over all $h \in \mathfrak{L}_{\mathfrak{B}^{p_{0}},\left(p(\cdot) / p_{0}\right)^{\prime}}$ with $\|h\|_{\mathfrak{L B}_{u^{p}},\left(p(\cdot) / p_{0}\right)^{\prime}} \leq 1$, Theorem 4 , (17) and (18) yield the boundedness of the Calderón operator $S$ on $L M_{u}^{p(\cdot)}$.

We also use the technique from the extrapolation theory to study the mapping properties of the local sharp maximal functions, the geometrical maximal functions and the rough maximal functions on local Morrey spaces with variable exponents in [14]. The results in [14] rely on the boundedness of the Hardy-Littlewood maximal operator. Therefore, the results obtained in [14] are valid for local Morrey spaces with variable exponents with the exponent functions being globally log-Hölder continuous. Our results use the maximal function $N$. Therefore, in view of Theorems 1 and 3 , we just require $p(\cdot)$ to be log-Hölder continuous at origin and infinity for the boundedness of the Calderón operator on $L M_{u}^{p(\cdot)}$.

We give a concrete example for the weight function $u$ that satisfies the conditions in

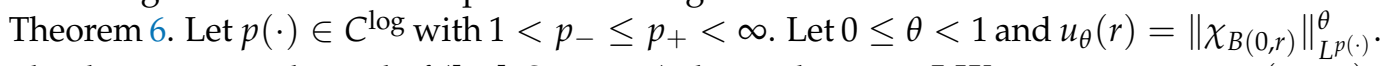
The discussion at the end of ([30], Section 2) shows that $u_{\theta} \in \mathbb{L} \mathbb{W}_{p(\cdot)}$. For any $p_{0} \in\left(1, p_{-}\right)$, we have

$$
u_{\theta}(r)^{p_{0}}=\left\|\chi_{B(0, r)}\right\|_{L^{p(\cdot)}}^{p_{0} \theta}=\left\|\chi_{B(0, r)}\right\|_{L^{p(\cdot) / p_{0}}}^{\theta} .
$$

The discussion at the end of ([30], Section 2) asserts that $u_{\theta}(r)^{p_{0}} \in \mathbb{L} \mathbb{W}_{p(\cdot) / p_{0}}$. Therefore, the conditions in Theorem 6 are fulfilled, and the Calderón operator $S$ is bounded on $L M_{u_{\theta}}^{p(\cdot)}$.

As $|\mathcal{H} f| \leq \mathcal{H}|f| \leq S|f|$ and $\left|\mathcal{H}^{*} f\right| \leq \mathcal{H}^{*}|f| \leq S|f|$, Theorem 6 yields the Hardy's inequalities on $L M_{u}^{p(\cdot)}$.

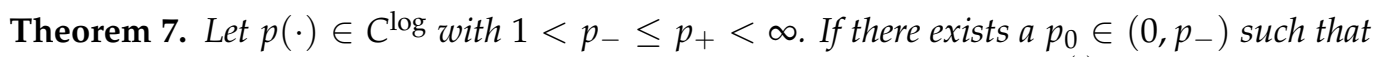
$u \in \mathbb{L} \mathbb{W}_{p(\cdot)}^{p_{0}}$, then there exists a constant $C>0$ such that for any $f \in L M_{u}^{p(\cdot)}$

$$
\begin{aligned}
\|\mathcal{H} f\|_{L M_{u}^{p(\cdot)}} & \leq C\|f\|_{L M_{u}^{p(\cdot)}}, \\
\left\|\mathcal{H}^{*} f\right\|_{L M_{u}^{p(\cdot)}} & \leq C\|f\|_{L M_{u}^{p(\cdot)}} .
\end{aligned}
$$

In particular, when $p(\cdot)=p, 1<p<\infty$ is a constant function, we have the Hardy's inequality on the local Morrey space $L M_{u}^{p}$. In addition, when $u \equiv 1$, the above results become the Hardy's inequalities on Lebesgue spaces with variable exponents, which recover the results in [31].

The reader is referred to $[2,18,19]$ for the history and applications of the Hardy' inequalities. For the Hardy's inequalities on the Hardy type spaces, the Lebesgue spaces with variable exponents and the Herz-Morrey spaces, the reader may consult [31-37].

Theorem 6 also yields the boundedness of the Stieltjes transformation, the RiemannLiouville and Weyl averaging operators on $L M_{u}^{p(\cdot)}$. 
Theorem 8. Let $p(\cdot) \in C^{\log }$ with $1<p_{-} \leq p_{+}<\infty$. If there exists a $p_{0} \in\left(0, p_{-}\right)$such that $u \in \mathbb{L} \mathbb{W}_{p(\cdot)}^{p_{0}}$, then there exists a constant $C>0$ such that for any $f \in L M_{u}^{p(\cdot)}$

$$
\begin{aligned}
\|H f\|_{L M_{u}^{p(\cdot)}} & \leq C\|f\|_{L M_{u}^{p(\cdot)}}, \\
\left\|I_{\alpha} f\right\|_{L M_{u}^{p(\cdot)}} & \leq C\|f\|_{L M_{u}^{p(\cdot)}}, \\
\left\|J_{\alpha} f\right\|_{L M_{u}^{p(\cdot)}} \leq C\|f\|_{L M_{u}^{p(\cdot)}} &
\end{aligned}
$$

The boundedness of the Stieltjes transformation on Lebesgue space is called as the Hilbert inequality. Therefore, as special cases of the preceding theorem, we also have the Hilbert inequality and the boundedness of the Riemann-Liouville and Weyl averaging operators on the local Morrey spaces $L M_{u}^{p}$ and the Lebesgue spaces with variable exponents $L^{p(\cdot)}$.

\section{Discussion}

We establish the boundedness of the Calderón operator on local Morrey spaces with variable exponents by extending the extrapolation theory. The exponent functions used in the local Morrey spaces with variable exponents are required to be log-Hölder continuous at the origin and infinity only. We need to refine the extrapolation theory for the maximal operator $N$ and the class of weight functions $A_{p, 0}$. In addition, in order to get rid of the approximation argument, we need to establish the embedding (16).

As applications of the main result, we have Hardy's inequalities, the Hilbert inequalities and the boundedness of the Riemann-Liouville and Weyl averaging operators on local Morrey spaces with variable exponents.

Moreover, we see that whenever we can establish the weighted norm inequalities with the class of weight function $A_{p, 0}$ for an operator $T$, even if $T$ is nonlinear, we can apply our extrapolation theory to obtain the boundedness of $T$ on the local Morrey spaces with variable exponents where the exponent function is log-Hölder continuous at 0 and infinity.

\section{Conclusions}

We extend the extrapolation theory to the local Morrey spaces with variable exponents with the exponent functions being log-Hölder continuous at the origin and infinity only. With this refined extrapolation theory, we obtain Hardy's inequalities and the Hilbert inequalities on the local Morrey spaces with variable exponents. Furthermore, the boundedness of the Calderón operator, the Riemann-Liouville operators and the Weyl averaging operators has been extended to the local Morrey spaces with variable exponents.

In particular, we have the Hardy's inequalities, the Hilbert inequalities on local Morrey spaces and the boundedness of the Calderón operator, the Riemann-Liouville averaging operators and the Weyl averaging operators on local Morrey spaces.

In conclusion, the results obtained in this paper generalize the existing results on the studies of local Morrey spaces with variable exponent, the Hardy's inequalities, the Hilbert inequalities on local Morrey spaces and the boundedness of the Calderón operator, the Riemann-Liouville averaging operators and the Weyl averaging operators.

Funding: This research was funded by The Education University of Hong Kong, Additional Reserach Fund R6391.

Institutional Review Board Statement: Not applicable.

Informed Consent Statement: Not applicable.

Conflicts of Interest: The author declares no conflict of interest. 


\section{References}

1. Bennett, C.; Sharpley, R. Interpolations of Operators; Academic Press: Cambridge, MA, USA, 1988.

2. Hardy, G.; Littlewood, J.; Pólya, G. Inequalities; Cambridge University Press: Cambridge, UK, 1952.

3. Duoandikoetxea, J.; Martin-Reyes, F.; Ombrosi, S. Calderón weights as Muckenhoupt weights. Indiana Univ. Math. J. 2013, 62, 891-910. [CrossRef]

4. Cruz-Uribe, D.; Dalmasso, E.; Martin-Reyes, F.; Ortega Salvador, P. The Calderón operator and the Stieltjes transform on variable Lebesgue spaces with weights. Collect. Math. 2020, 71, 443-469. [CrossRef]

5. Morrey, C.B. On the solutions of quasi-linear elliptic partial differential equations. Trans. Am. Math. Soc. 1938, 43, 126-166. [CrossRef]

6. Cruz-Uribe, D.; Fiorenza, A. Variable Lebesgue Spaces; Birkhäuser: Basel, Switzerland, 2013.

7. Diening, L.; Harjulehto, P.; Hästö, P.; Ružička, M. Lebesgue and Sobolev Spaces with Variable Exponents; Springer: Berlin/Heidelberg, Germany, 2011.

8. Burenkov, V.I.; Guliyev, H.V. Necessary and sufficient conditions for boundedness of the maximal operator in local Morrey-type spaces. Studia Math. 2004, 163, 157-176. [CrossRef]

9. Burenkov, V.I.; Guliyev, H.V.; Guliyev, V.S. Necessary and sufficient conditions for boundedness of the fractional maximal operator in the local Morrey-type spaces. J. Comp. Appl. Math. 2007, 208, 280-301. [CrossRef]

10. Burenkov, V.I.; Guliyev, V.S.; Tararykova, T.V.; Serbetci, A. Necessary and sufficient conditions for the boundedness of genuine singular integral operators in Local Morrey-type spaces. Dokl. Akad. Nauk 2008, 422, 11-14. [CrossRef]

11. Burenkov, V.; Gogatishvili, A.; Guliyev, V.S.; Mustafayev, R. Boundedness of the fractional maximal operator in local Morrey-type spaces. Compl. Variabl. Ellipt. Equat. 2010, 55, 739-758. [CrossRef]

12. Burenkov, V.; Nursultanov, E. Description of interpolation spaces for local Morrey-type spaces. Proc. Steklov Inst. Math. 2010, 269, 46-56. [CrossRef]

13. Guliyev, V.S. Generalized local Morrey spaces and fractional integral operators with rough kernel. J. Math. Sci. 2013, 193, 211-227. [CrossRef]

14. Yee, T.-L.; Cheung, K.L.; Ho, K.-P.; Suen, C.K. Local sharp maximal functions, geometrical maximal functions and rough maximal functions on local Morrey spaces with variable exponents. Math. Inequal. Appl. 2020, 23, 1509-1528. [CrossRef]

15. Rubio de Francia, J. Factorization and extrapolation of weights. Bull. Am. Math. Soc. 1982, 7, 393-395. [CrossRef]

16. Rubio de Francia, J. A new technique in the theory of $A_{p}$ weights. In Topics in Modern Harmonic Analysis, Vol. I, II (Turin/Milan, (1982); Istituto Nazionale di Alta Matematica Francesco Severi: Rome, Italy, 1983; p. 571579.

17. Rubio de Francia, J. Factorization theory and $A_{p}$ weights. Am. J. Math. 1984, 106, 533-547. [CrossRef]

18. Kufner, A.; Maligranda, L.; Persson, L.-E. The prehistory of the Hardy inequality. Am. Math. Mon. 2006, 113, 715-732. [CrossRef]

19. Kufner, A.; Persson, L.-E.; Samko, N. Weighted Inequalities of Hardy Type; World Scientific Publishing Company: Singapore, 2017.

20. Andersen, K. Weighted inequalities for the Stieltjes transformation and Hubert's double series. Proc. R. Soc. Edinb. Sect. A 1980, 86, 75-84. [CrossRef]

21. Gogatishvili, A.; Kufner, A.; Persson, L.-E. The weighted Stieltjes inequality and applications. Math. Nachr. 2013, 286, 659-668. [CrossRef]

22. Gogatishvili, A.; Persson, L.-E.; Stepanov, V.; Wall, P. Some scales of equivalent conditions to characterize the Stieltjes inequality: The case $q<p$. Math. Nachr. 2014, 287, 242-253.

23. Bastero, J.; Milman, M.; Ruiz, F. On the connection between weighted norm inequalities, commutators and real interpolation. In Memoirs of the American Mathematical Society; American Mathematical Society: Providence, RI, USA, 2001 ; Volume 154.

24. Almeida, A.; Drihem, D. Maximal, potential and singular type operators on Herz spaces with variable exponents. J. Math. Anal. Appl. 2012, 394, 781-795. [CrossRef]

25. Ho, K.-P. Extrapolation to Herz spaces with variable exponents and applications. Rev. Mat. Complut. 2020, 33, 437-463. [CrossRef]

26. Ho, K.-P. Spherical maximal function, maximal Bochner-Riesz mean and geometrical maximal function on Herz spaces with variable exponents. Rend. Circ. Mat. Palermo Ser. II 2021, 70, 559-574 . [CrossRef]

27. Ho, K.-P. Singular integral operators and sublinear operators on Hardy local Morrey spaces with variable exponents. Bull. Sci. Math. 2021, 171, 103033.

28. Sawano, Y.; Ho, K.-P.; Yang, D.; Yang, S. Hardy spaces for ball quasi-Banach function spaces. Diss. Math. 2017, 525, 1-102. [CrossRef]

29. Tao, J.; Yang, D.; Yuan, W.; Zhang, Y. Compactness Characterizations of Commutators on Ball Banach Function Spaces. Potential Anal. 2021. [CrossRef]

30. Ho, K.-P. Definability of singular integral operators on Morrey-Banach spaces. J. Math. Soc. Jpn. 2020, 72, 155-170. [CrossRef]

31. Diening, L.; Samko, S. Hardy inequality in variable exponent Lebesgue spaces. Fract. Calc. Appl. Anal. 2007, 10, 1-18.

32. Andersen, K.; Sawyer, E. Weighted norm Inequalities for the Riemann-Liouville and Weyl fractional integral operators. Trans. Am. Math. Soc. 1988, 308, 547-558. [CrossRef]

33. Cruz-Uribe, D.; Mamedov, F. On a general weighted Hardy type inequality in the variable exponent Lebesgue spaces. Rev. Mat. Complut. 2012, 25, 335-367. [CrossRef]

34. Ho, K.-P. Hardy's inequalities, Hilbert inequalities and fractional integrals on function spaces of $q$-integral $p$-variation. Ann. Polon. Math. 2021, 126, 251-263. [CrossRef] 
35. Mamedov, F.; Harman, A. On a Hardy type general weighted inequality in spaces $L^{p(\cdot)}$. Integral Equ. Oper. Theory 2010, 66, 565-592. [CrossRef]

36. Mamedov, F.; Zeren, Y. On equivalent conditions for the general weighted Hardy type inequality in space $L^{p(\cdot)}$. Z. Anal. Anwend. 2012, 31, 55-74. [CrossRef]

37. Yee, T.-L.; Ho, K.-P. Hardy's inequalities and integral operators on Herz-Morrey spaces. Open Math. 2020, 18, 106-121. [CrossRef] 